Pacific Journal of Mathematic 


\section{A FIXED POINT THEOREM FOR CHAINED SPACES}

L. E. WARD, JR.

1. Introduction. There are a number of theorems in the literature of the following type: if a topological space is acyclic in the sense of containing no simple closed curve, and if other appropriate conditions are satisfied then the space has the fixed point property, that is, each continuous function $f$ of the space into itself admits a solution of the equation $x=f(x)$. For example, if the space is compact metric and locally connected (i.e., a dendrite) then it has the fixed point property. There are many generalizations of this theorem. Appropriate to this discussion are of those of Borsuk [1], Plunkett [2], Wallace [3], the author [5] and [6], and Young [8]. A common characteristic of these generalizations is their requirement, explicit or implicit, of rather strong unicoherence conditions. But it is clear that many relatively simple acyclic spaces possessing the fixed point property are not unicoherent. As an example consider the following sets in the Cartesian plane:

$$
\begin{aligned}
& A=\{(x, y): 0<x \leq 1, y=\sin (\pi / x)\}, \\
& B=\{(0, y):-2 \leq y \leq 1\}, \\
& C=\{(x,-2): 0 \leq x \leq 1\}, \\
& D=\{(1, y):-2 \leq y \leq 0\} .
\end{aligned}
$$

The continuum $M=A \cup B \cup C \cup D$ is not unicoherent but it is arcwise connected, acyclic, and has the fixed point property. It is the purpose of this note to formulate and prove a fairly general result which includes this and related examples. In so doing we shall generalize the theorems of Borsuk and Young cited above. As in our earlier papers the methods used here are order-theoretic in character. Section 2 is devoted to the partial order stucture of the spaces to be considered, and may be regarded as an addendum to [4], [6] and [7].

2. Chained spaces. Throughout all spaces to be considered are Hausdorff. By a topological chain or, more simply, a chain, we mean a continuum (=compact connected set) which has exactly two non-cutpoints. These two points are, of course, endpoints and a chain is simply the natural analogue of an arc in spaces which are not assumed to be metric. A space is topologically chained or chained provided each two distinct points lie in some chain. Obviously each two distinct points of a chained space are the endpoints of some chain. If a space has the property that each two distinct points are the endpoints of at most one chain, then it is said to be acyclic. In this case the unique chain whose

Received February 4, 1959. 
endpoints are $x$ and $y$ is denoted $[x, y]$. It is convenient to define $[x, x]$ to be the set whose only element is $x$.

Acyclic chained spaces have an inherent partial order structure which facilitates their study. By a partial order on a set we mean a binary, reflexive, transitive relation $\leqq$ between elements of the set which, in addition, satisfies the rule

$$
x \leqq y \text { and } y \leqq x \text { implies } x=y .
$$

If $x \leqq y$ but $x \neq y$ we write $x<y$, and if $P$ is a partially ordered set we define

$$
L(x)=\{y \in P: y \leqq x\}, \quad M(x)=\{y \in P: x \leqq y\} .
$$

In order to characterize acyclic chained spaces we recall a related theorem from [7]. A dendritic space is a connected and locally connected space in which each two distinct points can be separated by the omission of some third point.

THEOREM 1. A necessary and sufficient condition that a locally connected space be dendritic is that it admit a partial order satisfying

(i) $L(x)$ and $M(x)$ are closed sets for each point $x$,

(ii) if $x<y$ then there exists $z$ such that $\times<z$ and $z<y$,

(iii) for each $x$ and $y$ the set $L(x) \cap L(y)$ is nonempty, compact and simply ordered,

(iv) for each $x$ the set $M(x)-x$ is open.

Although many chained spaces are not locally connected (e.g., the space $M$ of $\S 1$ ) they can be made locally connected by properly altering the topology. This change of topology preserves the original chain structure of the space, and functions which are continuous in the orginal topology remain continuous in the new one. This technique appears to have originated with Young [8]. If $X$ is a Hausdorff space let us say that a chain component of $X$ is any subset of $X$ which is maximal with respect to being chained. The chain topology is that topology which results from taking the chain components of open sets of the given topology as a basis for the chain topology. It is easily seen (and was proved in [8]) that any space is locally connected in its chain topology.

LEMMA 1. An acyclic chained space is dendritic with respect to its chain topology.

Proof. Let $x$ and $y$ be distinct points of the acyclic chained space $X$ and let $z \in[x, y]-x \cup y$. Since $X$ is acyclic no chain in $X-z$ contains both $x$ and $y$, and therefore $z$ separates $x$ and $y$ in the chain topology. Since $X$ is connected and locally connected in the chain topology 
it is dendritic.

From Theorem 1 and Lemma 1 we infer that each acyclic chained space is endowed with an intrinsic partial order structure which can aptly be called the chain cutpoint ordering. It can be described in the following way (compare with [7]). Select an element $e$ and define $x \leqq y$ if and only if $x \in[e, y]$. We now prove that the chain cutpoint ordering characterizes the acyclic spaces.

THEOREM 2. A necessary and sufficient condition that the Hausdorff space $X$ be acyclic and chained is that it be dendritic in its chain topology.

Proof. The necessity was established in Lemma 1. To prove the sufficiency of the condition let $X$ be a space which is dendritic in its chain topology. By Theorem $1 X$ admits a partial order which satisfies (i) - (iv) relative to the chain topology. If $x$ and $y$ are distinct points of $X$ then by (ii) and (iii) they are contained in a continum $L(x) \cup L(y)$ and by Theorem 3 of [7] that continum is a tree. Since a tree is chained, so is $X$. If two distinct chains $C_{1}$ and $C_{2}$ have common endpoints, let $A_{1}$ be a component of $C_{1}-C_{2}, x$ and $y$ the endpoints of $\overline{A_{1}}$, and $\overline{A_{2}}$ the minimal subchain of $C_{2}$ which joins $x$ and $y$. Obviously no point can separate $x$ and $y$ in the chain topology, for it would have to lie in $A_{1} \cap \overline{A_{2}}=0$. Since this is a contradiction we conclude that $X$ is acyclic.

3. A condition on rays. Let $X$ be a space and $e \in x$. A ray of $X$ with endpoint $e$ is the union of a maximal nest of chains which have $e$ as a common endpoint. Thus, in a Euclidean space a half line emanating from the origin is a ray in this sense. In the example of $\S 1$ the set $A$ is a ray of $M$ with endpoint $(1,0)$.

If $R$ is a ray with endpoint $e$ in the space $X$ and $x \in R$, let $A(R, x)$ be the closure of $(R-[e, x]) \cup x$, We then define

$$
K_{R}=\bigcap\{A(R, x): x \in R\} .
$$

In a Euclidean space a ray $R$ consisting of a half line emanating from the origin has $K_{R}=0$. However, in the example of $\S 1$ the set $A$ has $K_{A}$ equal to a closed line segment.

The crux of our fixed point argument is the following. If $f: X \rightarrow X$ is continuous where $X$ is acyclic and chained, we examine the points $x$ such that $x \leqq f(x)$. Either there is a "last" such point in a restricted order-theoretic sense, in which case that point is fixed by a continuity argument, or else such points form a ray $R$. Then we can show that $f\left(K_{R}\right) \subset K_{R}$, so that the fixed point property follows provided each $K_{R}$ has the fixed point property. 
We begin by formalizing this condition on rays.

$\left(F_{A}\right)$ If $R$ is a ray with endpoint a then $K_{R}$ has the fixed point property.

In the example of $\S 1$ let $a=(1,-2)$. Then there are two rays with endpoint $a, B \cup C$ and $A \cup D$. Since $K_{B \cup O}$ is a point and $K_{A \cup D}$ is a line segment the space $M$ satisfies $\left(F_{a}\right)$.

THEOREM 3. If $X$ is an arcwise connected space in which the union of any nest of arcs is contained in an arc then $X$ is acyclic and $X$ satisfies $\left(F_{a}\right)$ for each $a \in X$.

Proof. Since the union of any nest of arcs is contained in an arc, $X$ is acyclic; and if $R$ is a ray then $\bar{R}$ is evidently an arc so that $K_{R}$ is a point.

The substance of Young's fixed point theorem [8] is that the spaces of Theorem 3 have the fixed point property; hence, Theorem 5 below is truly a generalization.

THEOREM 4. If $X$ is an arcwise connected, hereditarily unicoherent continuum then $X$ satisfies $\left(F_{a}\right)$ for each $a \in X$.

Proof. We note that each subcontinuum of $X$ is arcwise connected, for if $x$ and $y$ are elements of the subcontinuum $Y$ and $[x, y]-Y$ is not empty then $[x, y] \cup Y$ would not be unicoherent. Now if $R$ is a ray of $X$ then $K_{R}$, being the intersection of a nest of continua, is a continuum and hence is itself arcwise connected and hereditarily unicoherent. Borsuk's theorem [1] asserts that such sets have the fixed point property.

This result demonstrates that all continua statisfying the hypothesis of Borsuk's fixed point theorem are included in Theorem 5 .

If $A$ and $B$ are subsets of a partially ordered set with $A \subset B$ then $A$ is cofinal in $B$ provided for each $b \in B$ there exists $a(b) \in A$ such that $b \leqq a(b)$.

Theorem 5. Let $X$ be a topologically chained acyclic space and suppose there exists $e \in X$ such that $\left(F_{e}\right)$ is satisfied. Then $X$ has the fixed point property.

Proof. We give $X$ the chain cutpoint ordering with minimal element $e$ and let $f: X \rightarrow X$ be a continuous function. Consider the family $\mathscr{S}$ of all pairs $\left(S, S^{\prime}\right)$ satisfying the following six conditions :

(i) $S$ is a nonempty simply ordered subset of $X$, 
(ii) $S$ and $S^{\prime}$ are connected,

(iii) $S^{\prime}$ is cofinal in $S$,

(iv) $\mathrm{e} \in S$,

(v) $x \leqq f(x)$ for each $x \in S$,

(vi) $S \cup f\left(S^{\prime}\right)$ is simply ordered.

Obviously the pair $(e, e)$ is a member of $\mathscr{S}$. We can partially order $\mathscr{S}$ by defining $\left(S_{\gamma}, S_{\gamma}^{\prime}\right) \prec\left(S_{\delta}, S_{\delta}^{\prime}\right)$ if and only if $S_{\gamma} \subset S_{\delta}$ and $S_{\delta} \cup \mathrm{f}\left(S_{\gamma}^{\prime}\right)$ is simply ordered. If $\mathscr{N}=\left\{\left(S_{\gamma}, S^{\prime}{ }_{\gamma}\right)\right\}$ is a $\prec-$ simple subfamily of $\mathscr{S}$ and $S=\bigcup\left\{S_{\gamma}\right\}, S^{\prime}=\bigcup\left\{S_{\gamma}^{\prime}\right\}$ then it is clear that $\left(S, S^{\prime}\right) \in \mathscr{S}$ and that $\left(S, S^{\prime}\right)$ is a $\prec-$ upper bound of $\mathscr{T}$. Thus Zorn's lemma can be applied; let $\left(S_{0}, S_{0}^{\prime}\right)$ be a $\prec-$ maximal member of $\mathscr{S}$.

If $x_{0}=\sup S_{0}$ exists we assert that $x_{0} \leqq f\left(x_{0}\right)$. For suppose there is $t \in S_{0}$ such that $f\left(x_{0}\right)$ is not a successor of $t$. We may assume $t<f(t)$; if $T=\left[t, x_{0}\right]$ then $f(T)$ is a tree and $t$ separates $f(t)$ and $f\left(x_{0}\right)$ in $f(T)$. If $W$ is the component of $f(T)-t$ which contains $f\left(x_{0}\right)$ then $W$ is a neighborhood of $f\left(x_{0}\right)$ in the relative topology of $f(T)$ and hence there is $q \in S_{0}, t<q<x_{0}$ such that $f(q) \in W$. But this implies that $f(q)$ is not a successor of $q$, a contradiction. Therefore, $t \leqq f\left(x_{0}\right)$ for each $t \in S_{0}$ and hence $x_{0} \leqq f\left(x_{0}\right)$. If $x_{0}<f\left(x_{0}\right)$ let $U$ be a connected neighborhood of $f\left(x_{0}\right)$ relative to the chain topology such that $\bar{U} \subset X-x_{0}$. Then there exists $x_{1} \in X-\bar{U}$ such that $x_{0}<x_{1}<f\left(x_{0}\right)$ and $f\left(\left[x_{0}, x_{1}\right]\right)$ $\subset U$. But then each point $p \in\left[x_{0}, x_{1}\right]$ satisfies $p \leqq f(p)$; letting $S_{1}=S_{0}$ $\cup\left[x_{0}, x_{1}\right], S_{1}^{\prime}=x_{1}$, it is apparent that $\left(S_{0}, S_{0}^{\prime}\right) \prec\left(S_{1}, S_{1}^{\prime}\right)$ in contradiction of the maximality of $S_{0}$. Hence $x_{0}=f\left(x_{0}\right)$.

On the other hand if $S_{0}$ has no supremum it is a ray $R$ with endpoint $e$ and it remains only to show that $f\left(K_{R}\right) \subset K_{R}$. By (vi) and the fact that $S_{0}=R$ is a ray we have $f\left(S_{0}^{\prime}\right) \subset R$. Moreover, $A(R, x) \subset \bar{S}_{0}^{\prime}$ for each $x \in S_{0}^{\prime}$ and hence $K_{R} \subset \overline{S_{0}^{\prime}}$. Therefore, $f\left(K_{R}\right) \subset \bar{R}$. Now suppose $f(y) \in \bar{R}-K_{R}$ for some $y \in K_{R}$. Let $V$ be a neighborhood of $f(y)$ such that $\bar{V}$ and $K_{R}$ are disjoint; then $\bar{V}$ and $A(R, x)$ are disjoint for some $x \in R$ and there exists $a \in R-[e, x]$ such that $f(a) \in V$. Moreover, it is clear by (ii) and (iii) that $a$ may be so chosen that $a$ $\in S_{0}^{\prime}$ and hence $f(\mathrm{a}) \in A(R, x)$, a contradiction. Therefore, $f\left(K_{R}\right) \subset K_{R}$ and the proof is complete.

\section{REFERENCES}

1. K. Borsuk, A theorem on fixed points, Bull. Acad. Polon. Sci. CI III, 2 (1954), 17-20.

2. R. L. Plunkett, A fixed point theorem for continuous multivalued transformations, Proc. Amer. Math. Soc., 7 (1956), 160-3.

3. A. D. Wallace, A fixed point theorem for trees, Bull. Amer. Math. Soc., 47 (1941), 757-60.

4. L. E. Ward, Jr., A note on dendrites and trees, Proc. Amer. Math. Soc., 5 (1954), $992-4$. 
5. —, Mobs, trees and fixed points, Proc. Amer. Math. Soc., 8 (1957), 798-804.

6. - A fixed point theorem for multi-valued functions, Pacific J. Math., 8 (1958), 921-927.

7. - On dendritic sets, Duke Math. J., 25 (1958), 505-14.

8. G. S. Young, The introduction of local connectivity by change of topology, Amer. J. Math., 68 (1946), 479-94.

\section{U. S. Naval ORdnance Test Station}

China LAKe, CALIFORNIA

UNIVERSITY OF OREGON 


\section{PACIFIC JOURNAL OF MATHEMATICS}

EDITORS

David Gilbarg

Stanford University

Stanford, California

R. A. Beaumont

University of Washington

Seattle 5 , Washington
A. L. Whiteman

University of Southern California

Los Angeles 7, California

L. J. PAIGE

University of California

Los Angeles 24, California

\section{ASSOCIATE EDITORS}

\author{
E. F. BECKENBACH \\ C. E. BURGESS \\ E. HEWITT \\ A. HORN
}

\author{
V. GANAPATHY IYER \\ R. D. JAMES \\ M. S. KNEBELMAN \\ L. NACHBIN
}
I. NIVEN
E. G. STRAUS
T. G. OSTROM
G. SZEKERES
H. L. ROYDEN
F. WOLF
M. M. SCHIFFER
K. YOSIDA

\section{SUPPORTING INSTITUTIONS}

\author{
UNIVERSITY OF BRITISH COLUMBIA \\ CALIFORNIA INSTITUTE OF TECHNOLOGY \\ UNIVERSITY OF CALIFORNIA \\ MONTANA STATE UNIVERSITY \\ UNIVERSITY OF NEVADA \\ OREGON STATE COLLEGE \\ UNIVERSITY OF OREGON \\ OSAKA UNIVERSITY \\ UNIVERSITY OF SOUTHERN CALIFORNIA
}

\author{
STANFORD UNIVERSITY \\ UNIVERSITY OF TOKYO \\ UNIVERSITY OF UTAH \\ WASHINGTON STATE COLLEGE \\ UNIVERSITY OF WASHINGTON \\ $* \quad * \quad *$ \\ AMERICAN MATHEMATICAL SOCIETY \\ CALIFORNIA RESEARCH CORPORATION \\ HUGHES AIRCRAFT COMPANY \\ SPACE TECHNOLOGY LABORATORIES
}

Mathematical papers intended for publication in the Pacific Journal of Mathematics should be typewritten (double spaced), and the author should keep a complete copy. Manuscripts may be sent to any one of the four editors. All other communications to the editors should be addressed to the managing editor, L. J. Paige at the University of California, Los Angeles 24, California.

50 reprints per author of each article are furnished free of charge; additional copies may be obtained at cost in multiples of 50 .

The Pacific Journal of Mathematics is published quarterly, in March, June, September, and December. The price per volume (4 numbers) is $\$ 12.00$; single issues, $\$ 3.50$. Back numbers are available. Special price to individual faculty members of supporting institutions and to individual members of the American Mathematical Society: $\$ 4.00$ per volume; single issues, $\$ 1.25$.

Subscriptions, orders for back numbers, and changes of address should be sent to Pacific Journal of Mathematics, 2120 Oxford Street, Berkeley 4, California.

Printed at Kokusai Bunken Insatsusha (International Academic Printing Co., Ltd.), No. 6, 2-chome, Fujimi-cho, Chiyoda-ku, Tokyo, Japan.

PUBLISHED BY PACIFIC JOURNAL OF MATHEMATICS, A NON-PROFIT CORPORATION

The Supporting Institutions listed above contribute to the cost of publication of this Journal, but they are not owners or publishers and have no responsibility for its content or policies. 


\section{Pacific Journal of Mathematics}

\section{Vol. 9, No. 4 \\ August, 1959}

Frank Herbert Brownell, III, A note on Kato's uniqueness criterion for

Schrödinger operator self-adjoint extensions ............... 953

Edmond Darrell Cashwell and C. J. Everett, The ring of number-theoretic

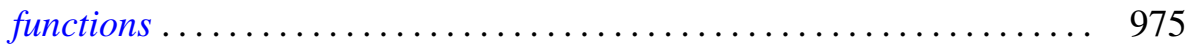

Heinz Otto Cordes, On continuation of boundary values for partial

differential operators ............................. 987

Philip C. Curtis, Jr., n-parameter families and best approximation . . . . . . 1013

Uri Fixman, Problems in spectral operators . . . . . . . . . . . . . . . 1029

I. S. Gál, Uniformizable spaces with a unique structure .............. 1053

John Mitchell Gary, Higher dimensional cyclic elements ............ 1061

Richard P. Gosselin, On Diophantine approximation and trigonometric

polynomials ..................................... 1071

Gilbert Helmberg, Generating sets of elements in compact groups ........ 1083

Daniel R. Hughes and John Griggs Thompson, The H-problem and the

structure of $H$-groups .................................. 1097

James Patrick Jans, Projective injective modules ................. 1103

Samuel Karlin and James L. McGregor, Coincidence properties of birth and

death processes ..................................... 1109

Samuel Karlin and James L. McGregor, Coincidence probabilities ........ 1141

J. L. Kelley, Measures on Boolean algebras ................... 1165

John G. Kemeny, Generalized random variables ................... 1179

Donald G. Malm, Concerning the cohomology ring of a sphere bundle ... . . 1191

Marvin David Marcus and Benjamin Nelson Moyls, Transformations on

tensor product spaces .................................. 1215

Charles Alan McCarthy, The nilpotent part of a spectral operator ........ 1223

Kotaro Oikawa, On a criterion for the weakness of an ideal boundary

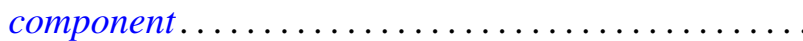

Barrett O'Neill, An algebraic criterion for immersion . . ............... 1239

Murray Harold Protter, Vibration of a nonhomogeneous membrane ... . . . . 1249

Victor Lenard Shapiro, Intrinsic operators in three-space . . . . . . . . . . . 1257

Morgan Ward, Tests for primality based on Sylvester's cyclotomic

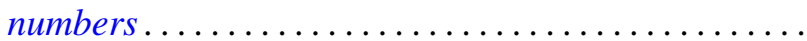

L. E. Ward, A fixed point theorem for chained spaces ....

Alfred B. Willcox, Šilov type $C$ algebras over a connected locally compact

abelian group..................................... 1279

Jacob Feldman, Correction to "Equivalence and perpendicularity of

Gaussian processes" ........................ 